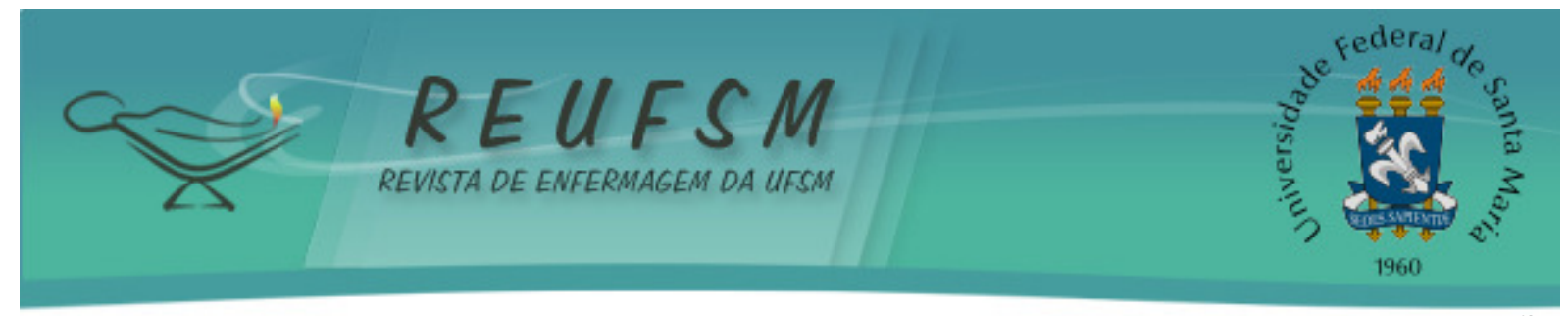

ARTIGO DE REVISÃO

\title{
OS SERVIÇOS DE SAÚDE SOB A ÓTICA DA ADOLESCENTE GRÁVIDA: UMA REVISÃO INTEGRATIVA DA LITERATURA
}

\author{
HEALTH SERVICES FROM THE PERSPECTIVE OF PREGNANT ADOLESCENTS: AN \\ INTEGRATIVE REVIEW OF THE LITERATURE
}

\section{LOS SERVICIOS DE SALUD DESDE LA PERSPECTIVA DE ADOLESCENTES EMBARAZADAS: UNA REVISIÓN INTEGRADORA DE LA LITERATURA}

\author{
Érica de Brito Pitilin ${ }^{1}$ \\ Carlos Alexandre Molena-Fernandes ${ }^{2}$ \\ Cláudio Claudino da Silva Filho ${ }^{3}$
}

\section{Doi: $10.5902 / 2179769215454$}

RESUMO: Objetivo: identificar na literatura científica a atuação dos serviços de saúde em relação à gravidez na adolescência sob a ótica da adolescente grávida. Método: tratou-se de uma revisão integrativa da literatura por meio da consulta em três bases de dados: Literatura Latino-Americana do Caribe em Ciências da Saúde (LILACS), Base de Dados Bibliográficos Especializada na Área de Enfermagem do Brasil (BDENF) e Medical Literature Analysis and Retrieval System Online (MEDLINE), pelos descritores "gravidez na adolescência", "percepção" e "serviços de saúde" no período de abril de 2014. Resultados: percebeu-se a prevalência de uma assistência tecnicista baseada nas necessidades físicas de cada adolescente decorrente do estado gravídico, seguida de uma assistência discriminatória e julgatória. Conclusão: pode-se observar que a assistência prestada à adolescente grávida é ainda carente de um olhar cuidadoso, de uma postura acolhedora, respaldada no apoio e na escuta, direcionada às características e demandas específicas desse público.

Descritores: Gravidez na adolescência; Percepção; Serviços de saúde.

ABSTRACT: Aim: to identify on scientific literature the performance of health services in relation to teenage pregnancy from the perspective of the pregnant teenager. Method: the study is based on an integrative literature review by consulting three databases: Latin American Caribbean Literature on Health Sciences (LILACS), Specialized Bibliographic Data Base at Area Nursing Brazil (BDENF) and Medical Literature Analysis and Retrieval System Online (MEDLINE), by the descriptors "teenage pregnancy ", "perception" and "health services" in April 2014. Results: mostly, there was_a technical assistance based on the

\footnotetext{
${ }^{1}$ Enfermeira. Mestre em Enfermagem pela Universidade Estadual de Maringá (UEM). Professora Assistente do Curso de Graduação em Enfermagem da Universidade Federal da Fronteira Sul (UFFS), Campus Chapecó-SC. Membro do Grupo de Estudo e Pesquisa Interdisciplinar Saúde e Cuidado (GEPISC) e do Núcleo de Estudos e Pesquisas Multidisciplinares em Políticas, Avaliação e Atenção em Saúde (NEPEMAAS). Chapecó, Santa Catarina, Brasil. E-mail: erica.pitilin@uffs.edu.br

2 Educador Físico. Doutor em Farmacologia pela Universidade Estadual de Maringá (UEM). Diretor de Pósgraduação da Universidade Estadual do Paraná (UNESPAR). Professor Permanente do Programa de Pósgraduação em Enfermagem da UEM. Líder do Núcleo de Estudos e Pesquisas Multidisciplinares em Políticas, Avaliação e Atenção em Saúde (NEPEMAAS). Paranavaí, Paraná, Brasil. E-mail: unespar.posgraduacao@gmail.com

3 Enfermeiro, Doutorando em Enfermagem pela Universidade Federal de Santa Catarina (UFSC) e Mestre em Enfermagem pela Universidade Federal da Bahia (UFBA). Professor Assistente da Universidade Federal da Fronteira Sul (UFFS), Campus Chapecó-SC. Integrante do Laboratório de Pesquisa e Tecnologia em Educação em Enfermagem e Saúde (EDEN/UFSC) e colaborador UNA SUS/UFSC em Atenção Básica para o Programa Mais Médicos e PROVAB. Pesquisador dos grupos/CNPq: GEPEGECE/UFFS, NESCO e EAI/UNIVASF, VSQV/UFBA. Chapecó, Santa Catarina, Brasil. Email: claudio.filho@uffs.edu.br
} 


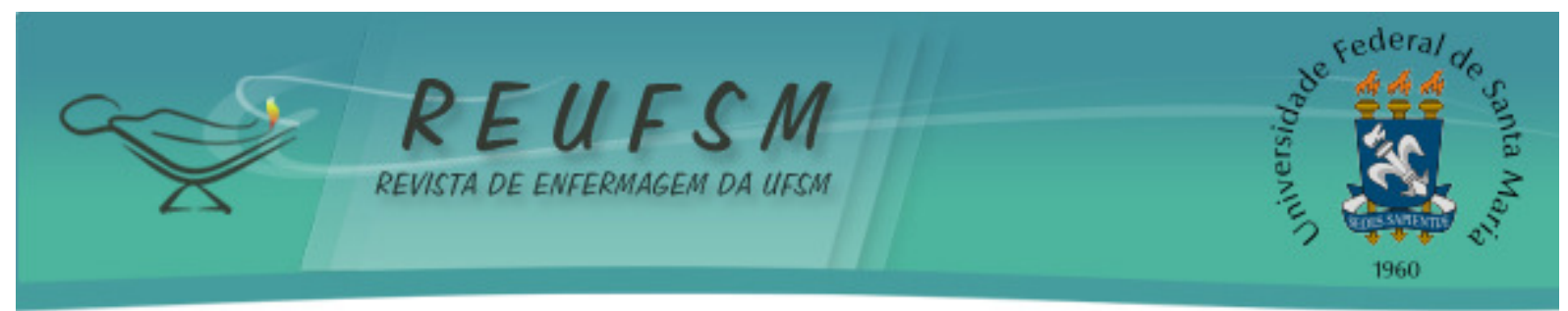

physical needs of each pregnant teenager, followed by a discriminatory and judgmental assistance. Conclusion: it can be noticed that the assistance provided to the pregnant teenager presents a lack of a careful overview and a welcoming attitude that aims to listen, support and fulfill every need of this type of patient.

Descriptors: Pregnancy in adolescence; Perception; Health services.

RESUMEN: Objetivo: identificar en la literatura científica el desempeño de los servicios de salud en el embarazo adolescente desde la perspectiva de la adolescente embarazada. Método: revisión integradora de la literatura mediante la consulta en tres bases de datos: el Caribe Latino Literatura Americana sobre Ciencias de la Salud (LILACS), Specialized Base de Datos Bibliográfica en el Área de Enfermería Brasil (BDENF) y Análisis de Literatura Médica y Recuperación del Sistema En Línea (MEDLINE), por los descriptores "embarazo en la adolescencia", "percepción" y "servicios de salud" en abril de 2014. Resultados: la prevalencia de una asistencia técnica en función de las necesidades físicas de cada embarazo adolescente, seguida de una asistencia discriminatorio y julgatória. Conclusión: la asistencia carece de una mirada cuidadosa, de una actitud de acogida, con el respaldo en el apoyo y escucha, dirigida a las características y necesidades de este público específico.

Descriptores: Embarazo en adolescencia; Percepción; Servicios de salud.

\section{INTRODUÇÃO}

A ocorrência da maternidade na adolescência constitui um fenômeno de repercussão mundial, cujo significado diverge nas diferentes culturas e contextos. Cerca de $11 \%$ de todos os nascimentos no mundo ocorrem em adolescentes entre 10 e 19 anos, e desses, $23 \%$ estão associados com morbidades decorrentes da gestação nessa faixa etária como prematuridade, baixo peso ao nascer e aumento dos índices de cesarianas. ${ }^{1}$ Ainda, acrescenta-se que a gravidez na adolescência é considerada um problema mundial de saúde pública uma vez que $64 \%$ dessas não são planejadas e $36 \%$ resultam em abortos ilegais, o que expressa o risco social e a magnitude de sua ocorrência. ${ }^{2}$

O Brasil tem apresentado declínio nos níveis de fecundidade a partir da década de 1970, mas a redução dessa taxa na adolescência ainda ocorre de maneira gradual. ${ }^{3}$ A literatura científica tem evidenciado a relevância da maternidade na adolescência que transcende além dos aspectos clínicos, sociais e econômicos, o impacto nos serviços de saúde, os quais não estão adequadamente preparados para receber e resolver as necessidades dessas jovens. ${ }^{4} \mathrm{O}$ uso de tais serviços depende de vários fatores abrangendo aspectos individuais, contextuais e a qualidade da assistência ofertada pelo profissional de saúde. ${ }^{5}$

Para os adolescentes o problema pode ser ainda maior, pois a procura espontânea pelos serviços é pequena ocorrendo, geralmente, quando já estão grávidas. ${ }^{6}$ Há certa dificuldade de implementação e estrutura do serviço público específico no atendimento à adolescente que acaba por ser atendida juntamente com a demanda geral da população. Os esforços realizados no sentido da criação de programas de qualidade tiveram, até certo ponto, resultados positivos por meio de um modelo de atendimento baseado na prestação da atenção integral ao adolescente. ${ }^{7}$ No entanto, a falta de privacidade e a inadequação de alguns programas voltados especificamente à essa clientela representam a principal razão para a dificuldade do acesso e a não utilização dos serviços de saúde pelo adolescente. ${ }^{2}$

A atenção ao adolescente, em especial à adolescente grávida, apresenta-se como um grande desafio pela ausência de adequação da linguagem e da forma de atuação das 


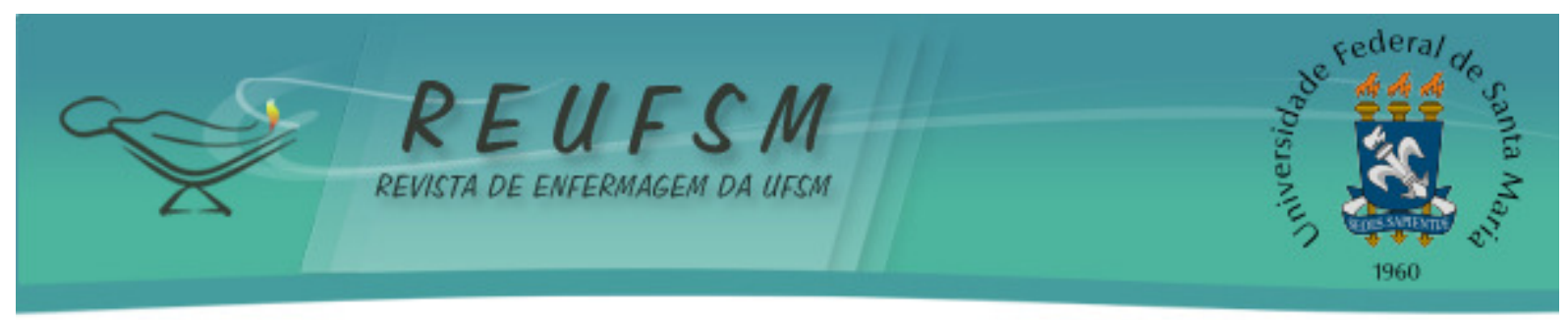

equipes de saúde. ${ }^{8}$ Sob essa ótica é necessário que estes profissionais estejam preparados para atender as necessidades e angustias geradas nesse complexo momento de transição entre a infância e a vida adulta, considerando não somente o caráter natural da sexualidade, mas entendendo-a como uma construção cultural. ${ }^{5}$ Além disso, devem conhecer os vários aspectos compreendidos na dinâmica familiar que abrange a adolescente grávida atuando como facilitadores e interlocutores nesse contexto.

A busca pela correspondência entre as perspectivas das adolescentes grávidas e as ações dos serviços de saúde constitui um elemento central de um trabalho socialmente relevante podendo fornecer subsídios para a reorganização da assistência direcionada à elas. Nesse sentido, questiona-se: "Como se dá a atuação dos serviços de saúde frente à problemática da gravidez na adolescência na perspectiva da adolescente grávida?" Dessa forma, este estudo teve por objetivo identificar na literatura científica a atuação dos serviços de saúde em relação à gravidez na adolescência sob a ótica da adolescente grávida, com o intuito de contribuir para a construção de novos conhecimentos sobre a temática, capaz de orientar as mudanças na assistência prestada.

\section{MÉTODO}

Trata-se de uma revisão integrativa da literatura, uma vez que esse método de pesquisa permite a busca, a avaliação crítica e a síntese das evidências disponíveis do tema investigado, sendo o seu produto final o estado atual do conhecimento do tema investigado, a implementação de intervenções efetivas na assistência à saúde e a redução de custos, bem como a identificação de lacunas que direcionam para o desenvolvimento de futuras pesquisas. ${ }^{9}$

Assim, visando à estruturação do estudo, delimitou-se as seguintes etapas propostas: definição da pergunta, estabelecimento de critérios para inclusão e exclusão dos estudos, definição das informações a serem extraídas dos estudos selecionados, avaliação dos estudos, interpretação dos resultados e, por fim, a síntese do conhecimento. ${ }^{9}$

A coleta de dados foi realizada por meio da consulta em três bases de dados: Literatura Latino-Americana do Caribe em Ciências da Saúde (LILACS), Base de Dados Bibliográficos Especializada na Área de Enfermagem do Brasil (BDENF) e Medical Literature Analysis and Retrieval System Online (MEDLINE). O levantamento dos estudos ocorreu no período de Abril de 2014. Para selecioná-los, foram utilizados os Descritores em Ciências da Saúde (DeCS) "gravidez na adolescência”, "percepção" e "serviços de saúde", permitindo que se realizasse uma busca avançada com os três descritores ao mesmo tempo pelo operador booleano "[AND]", da seguinte forma: "gravidez na adolescência and percepção and serviços de saúde".

Foram incluídos os estudos que responderam à questão de busca e que atenderam aos seguintes critérios: artigos originais de pesquisa, publicados em inglês, português ou espanhol e que estivessem disponíveis na íntegra online e gratuitos. Foram excluídas: publicações repetidas, cartas, editoriais, comentários, resumos de anais, teses, dissertações, trabalhos de conclusão de cursos, livros, cartas ao editor, estudos reflexivos, relatos de experiência, artigos de revisão bem como estudos que não abordassem a temática relevante ao objetivo da revisão.

A busca não teve seu tempo de publicação limitado por se tratar de um desenho de estudo específico, aumentando assim, sua força de evidência científica. Assim, por meio dos descritores citados a cima, foram encontrados 49 estudos, sendo 19 deles na base de dados MEDLINE, 17 na LILACS e 03 na BDENF. Após aplicação dos critérios de inclusão e exclusão foram analisados 13 artigos conforme apresentado na Figura 1. 


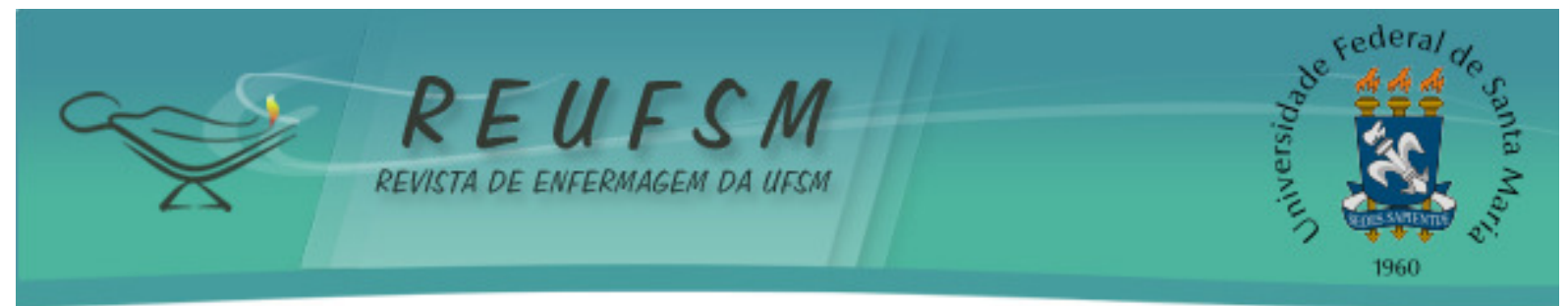

Para a seleção dos artigos, dois revisores independentes leram criticamente os títulos e os resumos das publicações identificadas e, em caso de dúvida ou discordância, um terceiro revisor foi solicitado a emitir parecer sobre a inclusão ou não do estudo. 0 grau de concordância entre os revisores foi estabelecido pela medida Kappa e o índice alcançado foi de $0,883 .{ }^{10}$ A avaliação desses artigos deu-se pela leitura e releitura na íntegra dos materiais selecionados com avaliação crítica e sistematização dos dados (Figura 1).

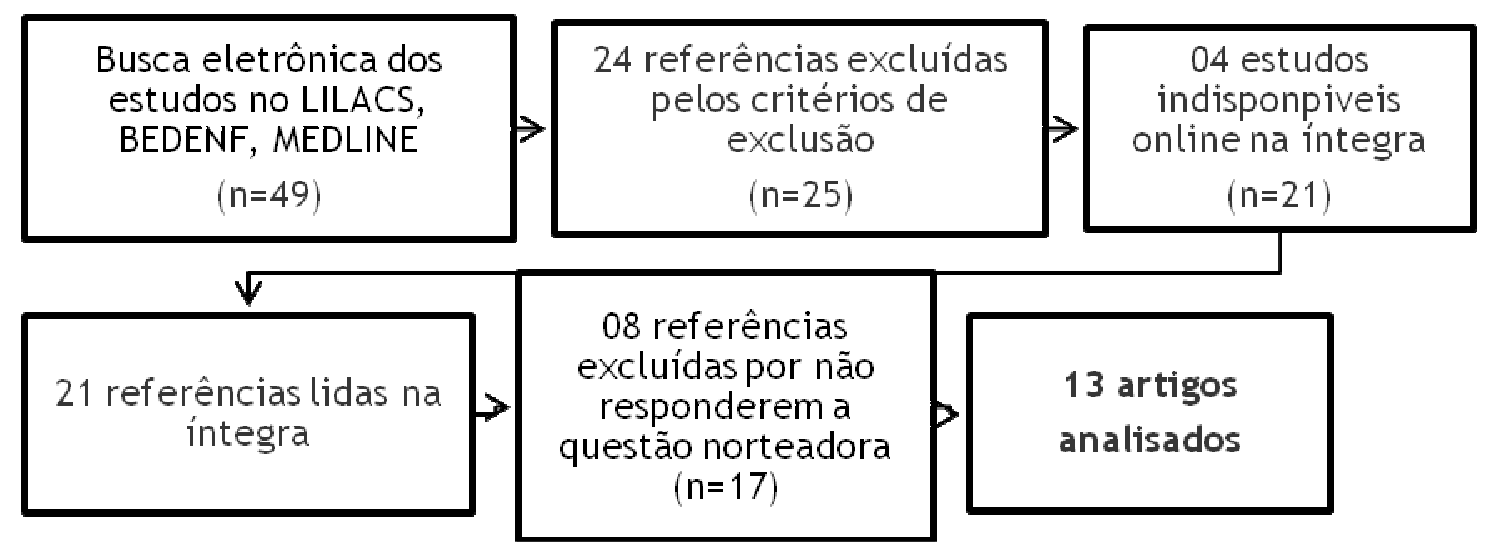

Figura 1- Fluxograma de identificação, busca, seleção e exclusão dos estudos para o desenvolvimento da pesquisa. Brasil, 2014.

Para a caracterização dos estudos selecionados cada artigo recebeu um código denominado pela letra A (artigo) seguido do número apresentado pela ordem da seleção na inclusão do estudo. Ressalta-se que, a partir da estratégia de busca de estudos pelas referências dos artigos selecionados, nenhum ensaio clínico foi encontrado. Assim, para a avaliação da qualidade metodológica que considera a produção do conhecimento científico na enfermagem em pesquisas desenvolvidas na abordagem qualitativa, utilizou-se os sistemas de classificação de evidências. ${ }^{11}$ Para a construção desse estudo foram respeitadas as dimensões éticas e as ideias centrais de cada um dos autores dos artigos analisados.

\section{RESULTADOS}

A caracterização das produções segundo ano, região, país, nível de evidência, abordagem do estudo, cenário do estudo e síntese dos resultados encontra-se no Quadro 1. Todos os artigos selecionados foram realizados no país, com predominância de pesquisas de abordagem qualitativa 13 (100\%). Além disso, 06 (46,1\%) trazem também a percepção dos familiares das adolescentes frente à assistência ofertada pelos serviços de saúde. Destacase a região Sudeste $06(46,1 \%)$ como maior evidência para a publicação dos estudos, seguida das regiões Nordeste 03 (23\%), Sul 03 (23\%) e Norte 01 (7,6\%). 0 cenário evidente quanto às publicações dos estudos foram as Unidades Básicas de Saúde $(57,1 \%)$. 


\begin{tabular}{|c|c|c|c|c|c|}
\hline Cód. & $\begin{array}{c}\text { Ano/ } \\
\text { Região/País }\end{array}$ & $\begin{array}{l}\text { *Nível } \\
\text { de } \\
\text { evidênc } \\
\text { ia }\end{array}$ & $\begin{array}{l}\text { Tipo de } \\
\text { Abordagem }\end{array}$ & $\begin{array}{l}\text { Cenário } \\
\text { do estudo }\end{array}$ & Síntese dos resultados \\
\hline $\mathrm{A} 1^{12}$ & $\begin{array}{c}\text { 2003/Nordeste/ } \\
\text { Brasil }\end{array}$ & 6 & Qualitativa & $\begin{array}{l}\text { Centro } \\
\text { médico }\end{array}$ & $\begin{array}{l}\text { Falta de informação ou de } \\
\text { acompanhamento. Baseada apenas no } \\
\text { físico. Assistência julgatória. }\end{array}$ \\
\hline $\mathrm{A} 2^{13}$ & $\begin{array}{l}\text { 2007/Norte/Bra } \\
\text { sil }\end{array}$ & 6 & Qualitativa & $\begin{array}{l}\text { Unidade } \\
\text { básica de } \\
\text { saúde }\end{array}$ & $\begin{array}{l}\text { Assistência discriminatória, tecnicista, } \\
\text { com falta de informações. }\end{array}$ \\
\hline $\mathrm{A} 3^{14}$ & $\begin{array}{l}\text { 2009/Sudeste/B } \\
\text { rasil }\end{array}$ & 6 & Qualitativa & $\begin{array}{l}\text { Unidade } \\
\text { básica de } \\
\text { saúde }\end{array}$ & $\begin{array}{l}\text { Questionamentos indevidos, ausência } \\
\text { de sigilo e informações. }\end{array}$ \\
\hline $\mathrm{A} 4^{15}$ & 2005/Sul/Brasil & 6 & Qualitativa & $\begin{array}{l}\text { Unidade } \\
\text { básica de } \\
\text { saúde }\end{array}$ & $\begin{array}{l}\text { Atendimento tecnicista. Práticas } \\
\text { burocráticas, falta de informações, } \\
\text { julgamento e pré-conceitos. }\end{array}$ \\
\hline$A 5^{16}$ & $\begin{array}{l}\text { 2010/Sudeste/B } \\
\text { rasil }\end{array}$ & 6 & Qualitativa & $\begin{array}{l}\text { Unidade } \\
\text { básica de } \\
\text { saúde }\end{array}$ & $\begin{array}{l}\text { Falta de humanização, despreparo } \\
\text { profissional em lidar com a situação. }\end{array}$ \\
\hline $\mathrm{A} 6^{17}$ & 1998/Sul/Brasil & 6 & Qualitativa & $\begin{array}{l}\text { Unidade } \\
\text { básica de } \\
\text { saúde }\end{array}$ & Indiferença, imparcialidade. \\
\hline$A 7^{18}$ & $\begin{array}{l}\text { 2008/Nordeste/ } \\
\text { Brasil }\end{array}$ & 6 & Qualitativa & $\begin{array}{l}\text { Unidade } \\
\text { básica de } \\
\text { saúde }\end{array}$ & $\begin{array}{l}\text { Atitudes preconceituosas, descaso com } \\
\text { a adolescente. }\end{array}$ \\
\hline $\mathrm{A} 8^{19}$ & 2012/Sul/Brasil & 6 & Qualitativa & $\begin{array}{l}\text { Unidade } \\
\text { básica de } \\
\text { saúde }\end{array}$ & $\begin{array}{c}\text { Imparcialidade na tentativa de prevenir } \\
\text { nova gravidez indesejada. Julgatória e } \\
\text { sem referência aos direitos } \\
\text { reprodutivos. }\end{array}$ \\
\hline$A 9^{20}$ & $\begin{array}{l}\text { 2009/Sudeste/B } \\
\text { rasil }\end{array}$ & 6 & Qualitativa & $\begin{array}{l}\text { Hospital } \\
\text { escola }\end{array}$ & Reprovação e punição. \\
\hline $\mathrm{A} 10^{21}$ & $\begin{array}{l}\text { 2003/Sudeste/B } \\
\text { rasil }\end{array}$ & 6 & Qualitativa & $\begin{array}{l}\text { Unidade } \\
\text { básica } \\
\text { de saúde }\end{array}$ & Práticas tecnicistas. \\
\hline $\mathrm{A} 11^{22}$ & $\begin{array}{l}\text { 2012/Sudeste/B } \\
\text { rasil }\end{array}$ & 6 & Qualitativa & $\begin{array}{l}\text { Hospital } \\
\text { escola }\end{array}$ & $\begin{array}{l}\text { Despreparo profissional em lidar com a } \\
\text { situação. }\end{array}$ \\
\hline $\mathrm{A} 12^{23}$ & $\begin{array}{c}\text { 2004/Nordeste/ } \\
\text { Brasil }\end{array}$ & 6 & Qualitativa & $\begin{array}{l}\text { Unidade } \\
\text { básica de } \\
\text { saúde }\end{array}$ & $\begin{array}{l}\text { Falta de informação e assistência } \\
\text { quanto ao planejamento da gravidez. }\end{array}$ \\
\hline $\mathrm{A} 13^{24}$ & $\begin{array}{l}\text { 2001/Sudeste/B } \\
\text { rasil }\end{array}$ & 6 & Qualitativa & $\begin{array}{l}\text { Unidade } \\
\text { básica } \\
\text { de saúde }\end{array}$ & $\begin{array}{l}\text { Dificuldade em abordar questões } \\
\text { relacionadas à sexualidade. }\end{array}$ \\
\hline
\end{tabular}

Quadro 1 - Características dos estudos selecionados na revisão integrativa segundo ano, região, país, nível de evidência, natureza do estudo, cenário do estudo e síntese dos resultados. Brasil, 2014.

Foi possível observar que em apenas $01(7,6 \%)$ artigo a adolescente se referia a atuação do enfermeiro em específico, nos demais, $12(92,4 \%)$ as práticas dos serviços de 


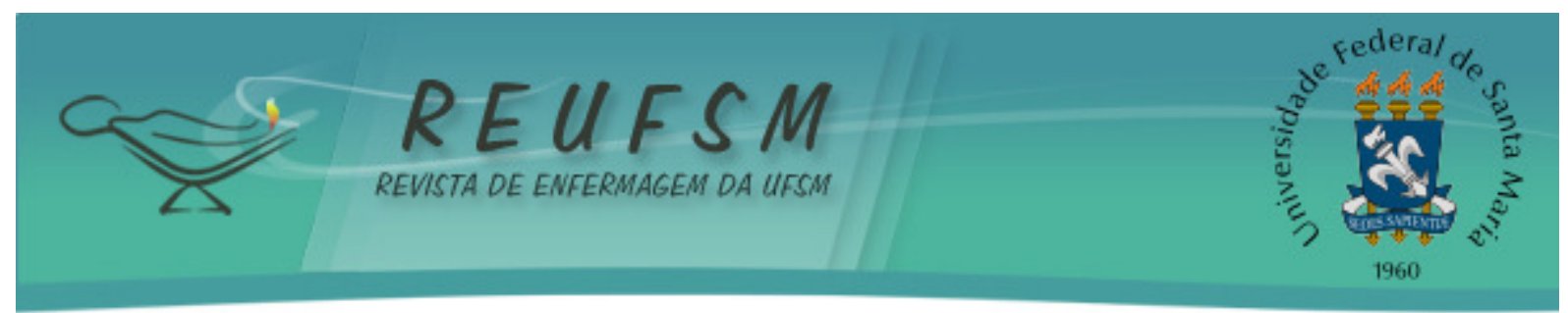

saúde foram elencadas como um todo, direcionando a atuação aos demais profissionais da saúde e a telefonista e recepcionista. Quanto à área de conhecimento, 03 (23\%) estudos eram da psicologia e o restante da área da enfermagem.

Devido à natureza do método encontrada nos estudos, não foi possível a análise estatística dos resultados encontrados. Para a análise dos dados, os mesmos foram estratificados de acordo com as unidades de interesse para o estudo. Tais unidades foram padronizadas e agrupadas conforme a similitude das ideias centrais apresentadas: como os serviços de saúde deveriam estar organizados e a abordagem das equipes de saúde sob a perspectiva da adolescente grávida.

\section{DISCUSSÃO}

Como se assinalou na introdução, a temática da gravidez na adolescência é relevante nas discussões no âmbito da saúde pública e na produção científica acadêmica da atualidade. A partir das informações obtidas por meio das análises dos dados foi possível enfocar a atuação dos serviços de saúde expondo as suas fragilidades. Com base nos estudos A9, A10 e A11 não há um planejamento específico das ações de saúde na prática para as adolescentes, gestantes ou não, havendo uma necessidade de intervenções voltadas à saúde sexual e reprodutiva nessa faixa etária. ${ }^{20,} 21,-22 \mathrm{Em}$ outras palavras, ainda persistem desigualdades no processo assistencial à adolescente na prática clínica.

A maneira como os serviços estão estruturados reflete na ausência de praticidade das ações voltadas ao planejamento familiar, o que representa uma dificuldade no acesso aos métodos contraceptivos existentes pela adolescente que necessita passar pela consulta médica, além da presença do responsável, inviabilizando a sua autonomia. ${ }^{6}$ Conforme assinalado no estudo $\mathrm{A} 8$, as unidades de saúde consistem apenas como um lugar burocrático de passagem para outros tipos de serviços, sendo utilizadas pelas adolescentes apenas durante o processo da gestação. ${ }^{19} \mathrm{~A}$ presença de um centro de saúde local com a distribuição livre de diferentes tipos de métodos contraceptivos não é o suficiente, uma vez que a falta de anonimato e o medo de divulgação de suas vidas sexuais corroboram com a não utilização dos serviços. ${ }^{3}$

Ainda quanto a atuação dos serviços de saúde, foi possível observar a ausência de ações programadas e de cunho educativo como assinalado no estudo $A 3 .{ }^{14} \mathrm{~A}$ falta de orientação encontrada nos estudos A4, A5 e A13 e a dificuldade das adolescentes em compreenderem a sua sexualidade e de aceitarem o seu novo corpo contribuíram para uma gravidez precoce e não planejada, uma vez que acreditavam que não engravidariam durante a primeira relação sexual. ${ }^{15-16,24} \mathrm{~A}$ falta de percepção sobre o risco de engravidar é o principal motivo para nunca haverem procurado os serviços de saúde antes da consumação do fato e da não utilização de métodos contraceptivos. ${ }^{1}$

Outro aspecto que pode ser observado a partir das análises dos dados foi como os serviços de saúde deveriam estar organizados. Os estudos A1 e A12 mostraram que é preciso o desenvolvimento de uma prática assistencial em saúde que vise não somente prevenir a gravidez, mas promover o uso de métodos contraceptivos, de modo a abordar os significados e as ansiedades que estão envolvidos nos diversos comportamentos da paquera, da atividade sexual e na transição entre a infância e a vida adulta. ${ }^{12,23}$ Trabalhar a interação entre os serviços de saúde e as escolas abordando tais direcionamentos por meio de atividades coletivas e individuais, bem como a criação de grupos específicos de gestantes adolescentes parecem ser relevantes no contexto da gravidez nesse grupo etário. ${ }^{25}$ Vale ressaltar que tais atividades devem ser elaboradas e desenvolvidas em uma linguagem própria direcionada a esse público, de maneira convidativa e entusiasmática e 


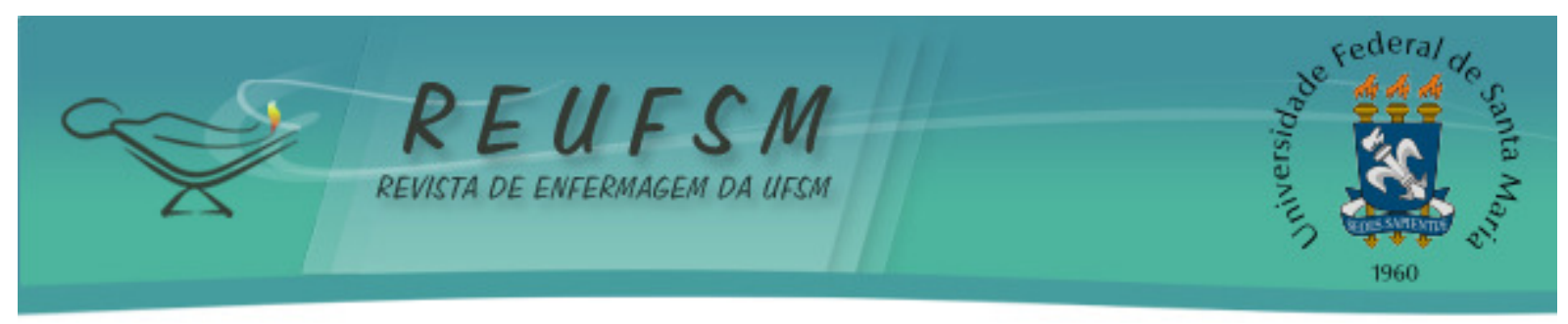

que possa ser claramente compreendida, encorajando a adolescente a apontar suas dúvidas e suas inseguranças. ${ }^{4}$

No que se refere à interação entre os serviços e os membros familiares os estudos A2, A6 e A7 apontam que não são trabalhadas questões relacionadas à sexualidade e ao ato sexual com os demais membros da família da adolescente. ${ }^{13,17-18}$ Os pais têm dificuldades e pudores em abordar tais assuntos direcionando essa responsabilidade para os serviços de saúde. A família necessita ser sujeito de uma atenção qualificada devendo constituir o eixo integrador das ações desenvolvidas pelas equipes. ${ }^{27}$ Desse modo, essa estratégia pode contribuir para a criação de um vínculo e a abertura de diálogos entre os membros da família para que se possa compartilhar angustias, dúvidas, curiosidades e anseios em relação à sexualidade, atividades sexuais e métodos contraceptivos, por exemplo. Uma relação familiar não harmoniosa, atribuída à dificuldade de comunicação/expressão, pode interferir posteriormente na continuidade da assistência a gestante adolescente durante a evolução da gravidez. ${ }^{5}$

Por fim, a abordagem das equipes de saúde sob a perspectiva da adolescente grávida evidenciada nos estudos A12 e A13 foi prevalente de uma assistência tecnicista baseada nas necessidades físicas de cada adolescente decorrente do estado gravídico, ${ }^{23-24}$ seguida de uma assistência discriminatória e julgatória, assinalada nos estudos A1 e A7. ${ }^{12,18}$ Tais medidas não contemplam o contexto global dessa adolescente repleta de transformações sociopsicológicas e anátomo-metabólicas à que está inserida. Evidencia-se a importância das ações dos profissionais em prevenir não somente a gravidez, mas também na adoção de medidas que abordem os significados e as ansiedades que estão envolvidos nos diversos comportamentos da paquera, atividade sexual e na transição entre a infância e a vida adulta. ${ }^{25}$

A vivência da sexualidade precisa ser percebida cada vez mais como algo positivo e natural, e não de maneira imoral e inibidora. Muitas vezes o profissional de saúde transforma a sexualidade em censura e condenação, o que acaba por inibir a jovem adolescente na aproximação aos serviços. ${ }^{13}$ Considera-se de extrema importância que os profissionais de saúde tenham conhecimento dos mitos que estão presentes no meio cultural das adolescentes e que podem influenciar em suas práticas de cuidado. ${ }^{26} \mathrm{Um}$ ponto relevante apontado por um dos estudos (A10) foi quanto a atuação do enfermeiro como intervencionista apenas no processo gestacional, não demonstrando interesse acerca da pessoa adolescente ou do seu problema em particular. ${ }^{21}$ Nesse contexto, a enfermagem possui um papel central na inserção de novas condutas e rotinas na prestação do cuidado à adolescente grávida, ao lidar diretamente com as demandas advindas desse público e por ser o coordenador do cuidado.

Assim, o que se observa é um consenso na literatura sobre a necessidade de reorganização no desenvolvimento das políticas públicas já existentes voltadas à demanda das reais necessidades das adolescentes no sistema de saúde. Os profissionais que estão à frente de tais serviços precisam atender integralmente as especificidades desta parcela da população de uma forma personalizada, focada na promoção da saúde.

\section{CONSIDERAÇÕES FINAIS}

Por meio do levantamento bibliográfico analisado pode-se observar que a assistência prestada à adolescente grávida é ainda carente de um olhar cuidadoso, de uma postura acolhedora, respaldada no apoio e na escuta, direcionada às características e demandas específicas desse público. Cabe aos profissionais de saúde refletir sobre o tipo de atenção que é dado à saúde do adolescente, aos seus direitos sexuais e reprodutivos. É preciso garantir subsídios para dialogar quanto ao planejamento das ações e dos serviços utilizados 


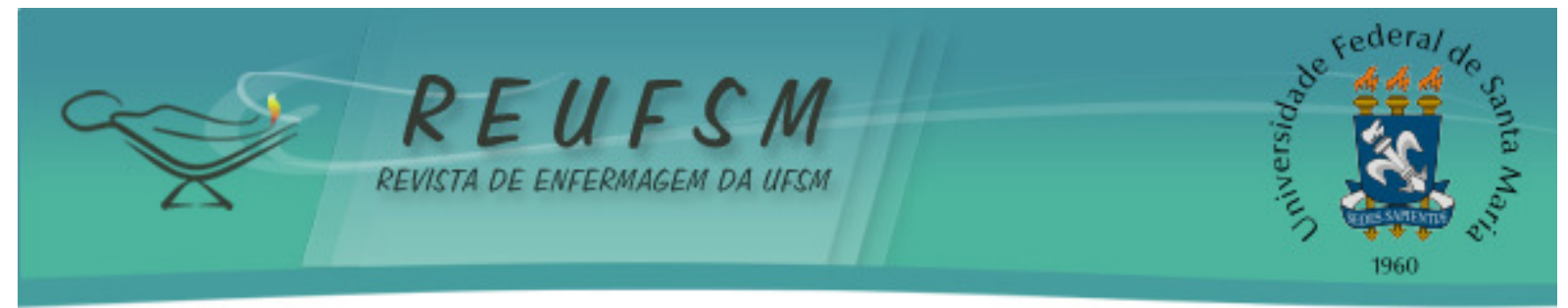

como estratégia para o atendimento nesse momento de transição, além de viabilizar o acesso do adolescente aos serviços de saúde e a participação familiar, neste contexto.

Diante do exposto, acredita-se que este estudo possa contribuir para uma reflexão e uma melhoria na atenção à saúde do adolescente prestada pelas equipes de saúde, em especial a enfermagem, uma vez que o enfermeiro atua como protagonista e condutor do gerenciamento da qualidade do serviço. Vale ressaltar que apenas um artigo trouxe a atuação do enfermeiro na perspectiva da adolescente grávida, o que pode resultar em uma lacuna na produção para esses profissionais. Cabe ao enfermeiro, chamar para si a responsabilidade de uma atenção livre de pré-conceitos que possam influenciar a qualidade da assistência prestada. Além disso, faz-se necessário entender o contexto que a adolescente está inserida diante do evento de uma gravidez em sua maioria não planejada.

Ademais, configura-se como uma limitação do estudo que foram incluídos apenas os artigos disponíveis de maneira gratuita via internet, tendo em vista que algum trabalho importante pode não ter sido considerado. Cabe ressaltar que os níveis de evidência identificados para a avaliação da qualidade metodológica da amostra dos artigos encontrados foram fracos, o que podem apontar lacunas na produção do conhecimento científico principalmente da área da enfermagem, visto que boa parte dos estudos selecionados para a amostra foram produzidos por enfermeiros. Assim, sugere-se que novas investigações de fortes evidências sejam realizadas no âmbito da saúde do adolescente diante desta temática.

\section{REFERÊNCIAS}

1. Santos NLAC, Costa MCO, Amaral MTR, Vieira GO, Bacelar EB, Almeida AHV. Gravidez na adolescência: análise de fatores de risco para baixo peso, prematuridade e cesariana. Ciênc Saúde Coletiva [Internet]. 2014 mar [acesso em 2014 abri 4];19(3):[08 páginas]. Disponível em: http://dx.doi.org/10.1590/1413-81232014193.18352013.

2. Daniels JP. Tackling teenage pregnancy in Colombia. Lancet [Internet]. $2015 \mathrm{abr}$ [acesso em 2015 maio 12];385(9977):[02 páginas]. Disponível em: http://www.thelancet.com/pdfs/journals/lancet/PIIS0140-6736(15)60738-3.pdf.

3. Santos K A. Teenage pregnancy contextualized: understanding reproductive intentions in a Brazilian shantytown. Cad Saúde Pública [Internet]. 2012 abr [acesso em 2014 jun 12];28(4):[14 screens]. Disponível em: http://dx.doi.org/10.1590/S0102$311 X 2012000400005$.

4. Ralph LJ, Brindis CD. Access to reproductive healthcare for adolescents: establishing healthy behaviors at a critical juncture in the lifecourse. Curr Opin Obstet Gynecol [Internet]. 2010 out [acesso em 2014 jun 12];22(5):[06 screens]. Disponível em: http://www.ncbi.nlm.nih.gov/pubmed/20733485.

5. Atuyambe L, Mirembe F, Tumwesigye NM, Annika J, Kirumira EK, Faxelid E. Adolescent and adult first time mothers health seeking practices during pregnancy and early motherhood in Wakiso district, central Uganda. Reprod Health [Internet]. 2008 dez [acesso em 2014 ago 21];5(13):[14 screens]. Disponível em: http://www.ncbi.nlm.nih.gov/pubmed/19116011.

6. Moura LNB, Gomes KRO. Planejamento familiar: uso dos serviços de saúde por jovens com experiência de gravidez. Ciênc Saúde Coletiva [Internet]. 2014 mar [acesso em 2014 jun 22];19(3):[10 páginas]. Disponível em: http://dx.doi.org/10.1590/141381232014193.10902013. 


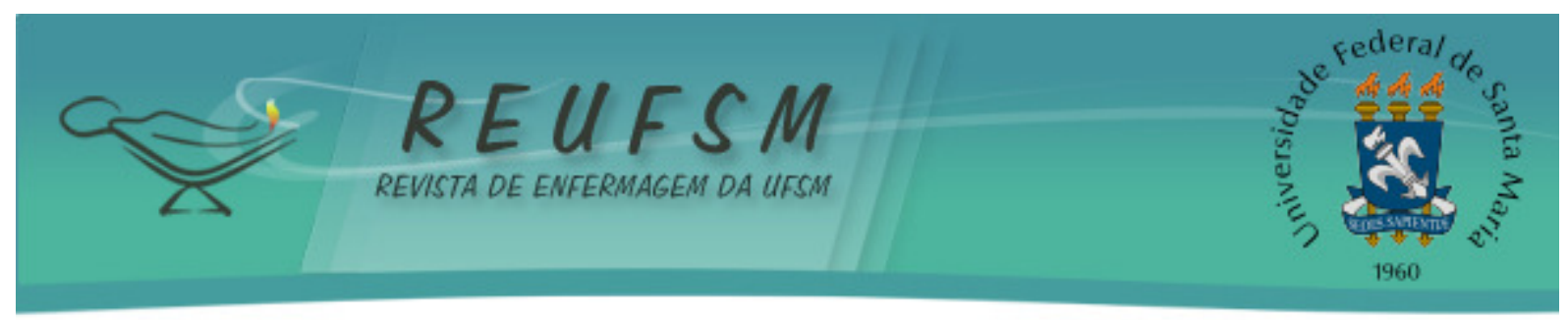

7. Brasil. Ministério da Saúde. Atenção à saúde do adolescente: competências e habilidades. Brasília (DF): Ministério da Saúde; 2008.

8. Malta DC, Silva MAI, Mello FCM, Monteiro RA, Porto DL, Sardinha LMV, et al. Saúde sexual dos adolescentes segundo a Pesquisa Nacional de Saúde dos Escolares. Rev Bras Epidemiol [Internet]. 2011 set [acesso em 2014 ago 12];14(1):[10 páginas]. Disponível em: http://dx.doi.org/10.1590/S1415-790X2011000500015.

9. Mendes KDS, Silveira RCCP, Galvão CM. Revisão integrativa: método de pesquisa para a incorporação de evidências na saúde e na enfermagem. Texto \& Contexto Enferm [Internet]. 2008 out [acesso em 2014 jun 21];17(4):[07 páginas]. Disponível em: http: //www.scielo.br/scielo.php?script=sci_arttext\&pid=S0104-07072008000400018.

10. Hulley SB, Cumming SR, Browner WS, Grady, DG, Newman, TB. Delineando a pesquisa clínica: uma abordagem epidemiológica. 3 ed. Porto Alegre: Artmed; 2008. 384 p.

11. Melnyk BM, Fineout-Overholt E. Making the case for evidence-based practice. In: Melnyk BM, Fineout-Overholt E. Evidence based practice in nursing and healthcare: a guide to best practice. Philadelphia: Lippincot Williams \& Wilkins; 2005. p. 3-24.

12. Silva DV, Salomão NMR. A maternidade na perspectiva de mãe adolescentes e avós maternas dos bebês. Estud Psicol [Internet]. 2003 jan-abr [acesso em 2014 jul 12];8(1):[10 páginas]. Disponível em: http://dx.doi.org/10.1590/S1413-294X2003000100015.

13. Pantoja FC, Bucher JSNF, Queiroz $\mathrm{CH}$. Adolescentes grávidas: vivências de uma nova realidade. Psicol Ciênc Prof [Internet]. 2007 set [acesso em 2014 ago 12];27(3):[11 páginas]. Disponível em http://www.scielo.br/scielo.php?pid=S141498932007000300011 \&script=sci_arttext.

14. Hoga LAK, Borges ALV, Alvarez REC. Gravidez na adolescência: valores e reações dos membros da família. Acta Paul Enferm [Internet]. 2009 set [acesso em $2014 \mathrm{abr}$ 12];22(6):[07 páginas]. Disponível em: http://dx.doi.org/10.1590/S010321002009000600009 .

15. Leal AC, Wall ML. Percepções da gravidez para adolescentes e perspectivas de vida diante da realidade vivenciada. Cogitare Enferm [Internet]. 2005 ago [acesso em 2014 jun 22];10(3):[09 páginas]. Disponível em: http://ojs.c3sl.ufpr.br/ojs/index.php/cogitare/article/view/5375.

16. Hoga LAK, Borges ALV, Reberte LM. Razoes e reflexos da gravidez na adolescência: narrativas dos membros da família. Esc Anna Nery Rev Enferm [Internet]. 2010 set [acesso em 2014 jun 12];14(1):[07 páginas]. Disponível em: http://www.scielo.br/pdf/ean/v14n1/v14n1a22.

17. Amazarray MR, Machado PS, Oliveira VZ, Gomes WB. A experiência de assumir a gestação na adolescência: um estudo fenomenológico. Psicol Reflex Crit [Internet]. 1998 jan-mar [acesso em 2014 abr 12];11(1):[13 páginas]. Disponível em: http: //dx.doi.org/10.1590/S0102-79721998000300004.

18. Moreira TMM, Viana DS, Queiroz MVO, Jorge MSB. Conflitos vivenciados pelas adolescentes com a descoberta da gravidez. Rev Esc Enferm USP [Internet]. 2008 jan-mar [acesso em 2014 jun 12];42(2):[09 páginas]. Disponível em: http: / / www.scielo.br/scielo.php?script=sci_arttext\&pid=S0080-62342008000200015.

19. Carvalho GM, Merighi MAB, Jesus MCP. Recorrência da parentalidade na adolescência na perspectiva dos sujeitos envolvidos. Texto \& Contexto Enferm [Internet]. 2009 set 


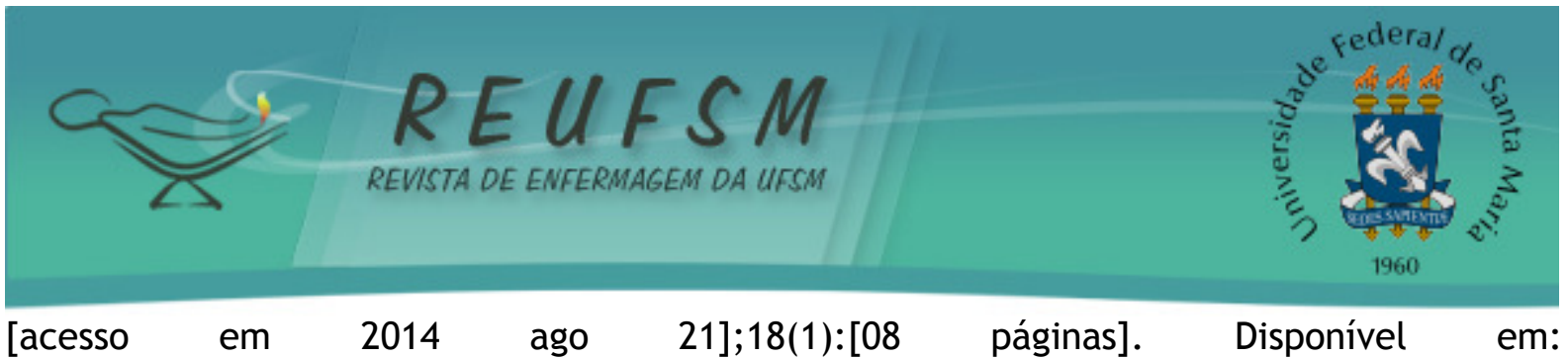
http://dx.doi.org/10.1590/S0104-07072009000100002.

20. Santos SR, Schor N. Vivências da maternidade na adolescência precoce. Rev Saúde Pública [Internet]. 2003 ago [acesso em 2014 jun 21];37(1):[09 páginas]. Disponível em: http://dx.doi.org/10.1590/S0034-89102003000100005.

21. Fernandes AO, Santos Junior HPO, Gualda DMR. Gravidez na adolescência: percepções das mães de gestantes jovens. Acta Paul Enferm [Internet]. 2012 mar-jun [acesso em 2014 jun 21];25(1):[10 páginas]. Disponível em: http: / /www.scielo.br/scielo.php?script=sci_arttext\&pid=S0103$21002012000100010 \&$ lng=pt\&nrm=iso\&tlng=pt.

22. Lima CT, Feliciano KVO, Carvalho MFS, Souza APP, Menabó JBC, Ramos LC, et al. Percepções e práticas de adolescentes grávidas e de familiares em relação à gestação. Rev Bras Saúde Matern Infant [Internet]. 2004 jan-mar [acesso em 2014 abr 21];4(1):[12 páginas]. Disponível em: http://dx.doi.org/10.1590/S1519-38292004000100007.

23. Gonçalves SD, Parada CMGL, Bertoncello NMF. Percepção de mães adolescentes acerca da participação paterna na gravidez, nascimento e criação do filho. Rev Esc Enferm USP [Internet]. 2001 set [acesso em $2014 \mathrm{abr}$ 21];35(4):[08 páginas]. Disponível em: http://www.scielo.br/pdf/reeusp/v35n4/v35n4a13.pdf.

24- Silva SC, Prates LA, Scarton J, Barreto CN, Alves CN, Wilhelm LA, et al. Mitos e dúvidas de adolescentes acerca das modificações corporais e suas implicações na sexualidade. Rev Enferm UFSM [Internet]. 2014 abr-jun [acesso em 2014 ago 22];4(2):[10 páginas]. Disponível em: http://cascavel.ufsm.br/revistas/ojs2.2.2/index.php/reufsm/article/view/10812.

25. Buendgens BB, Zampideri MF. A adolescente grávida na percepção de médicos e enfermeiros da atenção básica. Esc Anna Nery Rev Enferm [Internet]. 2012 mar [acesso em 2014 maio 4];16(1):[08 páginas]. Disponível em: http:/ / www.scielo.br/scielo.php?pid=S1414-81452012000100009\&script=sci_arttext.

26. Dias ACG, Teixeira MAP. Gravidez na adolescência: um olhar sobre um fenômeno complexo. Paidéia (Ribeirão Preto) [Internet]. 2010 mar [acesso em 2014 jun 12];20(45):[09 páginas]. Disponível em: http://dx.doi.org/10.1590/S0103$863 \times 2010000100015$.

27. Pitilin EB, Haracemiw A, Marcon SS, Pelloso SM. A família como sustentação no cotidiano de mulheres multíparas. Rev Gaúcha Enferm [Internet]. 2013 mar [acesso em 2015 maio 12];34(4):[07 páginas]. Disponível em: http://seer.ufrgs.br/index.php/RevistaGauchadeEnfermagem/article/view/40794/28550.

Data de recebimento: 05/01/2015

Data de aceite: 09/09/2015

Contato do autor responsável: Érica de Brito Pitilin

Endereço postal: Av General Osório - 480-D. 89802-210. Jardim Itália. Chapecó-Sc.

E-mail: erica.pitilin@uffs.edu.br 